\title{
Impurity transport in trapped electron mode driven turbulence
}

\author{
A. Mollén, ${ }^{1}$ I. Pusztai, ${ }^{1,2}$ T. Fülöp, ${ }^{1}$ and S. Moradi ${ }^{1}$ \\ ${ }^{1}$ Department of Applied Physics, Nuclear Engineering, Chalmers University of Technology and Euratom-VR \\ Association, Göteborg, Sweden \\ ${ }^{2}$ Plasma Science and Fusion Center, Massachusetts Institute of Technology, Cambridge, \\ Massachusetts 02139, USA
}

(Received 20 December 2012; accepted 5 March 2013; published online 28 March 2013)

\begin{abstract}
Trapped electron mode turbulence is studied by gyrokinetic simulations with the GYRO code and an analytical model including the effect of a poloidally varying electrostatic potential. Its impact on radial transport of high- $Z$ trace impurities close to the core is thoroughly investigated, and the dependence of the zero-flux impurity density gradient (peaking factor) on local plasma parameters is presented. Parameters such as ion-to-electron temperature ratio, electron temperature gradient, and main species density gradient mainly affect the impurity peaking through their impact on mode characteristics. The poloidal asymmetry, the safety factor, and magnetic shear have the strongest effect on impurity peaking, and it is shown that under certain scenarios where trapped electron modes are dominant, core accumulation of high- $Z$ impurities can be avoided. We demonstrate that accounting for the momentum conservation property of the impurity-impurity collision operator can be important for an accurate evaluation of the impurity peaking factor. (C) 2013 American Institute of Physics. [http://dx.doi.org/10.1063/1.4796196]
\end{abstract}

\section{INTRODUCTION}

Turbulence driven by unstable drift waves is considered to be responsible for most of the observed cross-field particle and heat transport in the core of tokamaks. In particular, ion gyro-radius scale drift waves destabilized by the non-adiabatic response of trapped electrons, the so-called trapped electron (TE) modes, can play an important role, specifically in conditions where the electron heating power is large compared to the ion heating power and the electron temperature is larger than the ion temperature. They can also be important in transport barrier regions, where the density gradient is large. ${ }^{1}$

Since its original discovery, ${ }^{2}$ TE modes have been the topic of theoretical investigations. They are usually categorized into the dissipative and collisionless classes. ${ }^{3}$ The dissipative TE mode requires a strong temperature gradient and large collisionality, while the collisionless TE mode, which is more likely to be destabilized in reactor relevant conditions, is driven by the electron curvature drift resonance and can be destabilized even in the absence of collisionality. The collisionless TE mode can be driven purely by the main species density gradient or by the electron temperature gradient. Consequently it is customary to further divide the collisionless TE mode into density gradient driven and electron temperature gradient driven categories. The stability and the turbulent fluxes driven by TE modes have been analyzed in Refs. 4 and 5. It has been shown in Refs. 5 and 6 that a quasilinear electrostatic approximation might retain much of the relevant physics of TE mode driven transport as it appears in nonlinear gyrokinetic simulations. The purpose of this paper is to study the impurity transport driven by TE modes.

It is well known that accumulation of impuritiesparticularly those with high charge number-in the core of fusion plasmas has debilitating effect on fusion reactivity due to radiative losses and plasma dilution. Results of fluid and gyrokinetic simulations ${ }^{7-23}$ indicated that the anomalous impurity transport driven by electrostatic microinstabilities, in general, and TE modes, in particular, is determined by the competition of three main mechanisms: curvature, thermodiffusion, and parallel compressibility. The first of these contributes to an inward impurity transport (when the magnetic shear is positive) while thermodiffusion depends on the direction of the mode propagation, being inward for modes propagating in the electron diamagnetic direction such as the TE modes; however, this contribution is negligible for high$Z$ impurities. The sign of the parallel compressibility contribution also depends on the direction of the mode propagation but is instead outward for TE modes and has a charge to mass ratio dependence.

In recent years attention has been directed towards the role of TE modes in impurity transport in plasmas with radio frequency $(\mathrm{RF})$ heating. Various experiments reported reduced impurity accumulation in such circumstances. ${ }^{24-26}$ In particular, it was shown in Ref. 27 that impurity transport was more affected by the change in the plasma parameters due to RF heating than by the generated sawtooth activity. In Refs. 7 and 8 it was argued that in a TE-dominated ASDEX-U discharge with Electron Cyclotron Resonance Heating (ECRH) the outward flows due to parallel compressibility explained the reduction in the impurity density peaking. In Ion Cyclotron Resonance Heating (ICRH) discharges on the JET tokamak the differences in the steady state impurity density profiles under minority heating (peaked impurity profiles) and mode conversion heating (hollow or flat impurity profiles) were partially explained by ITG and density gradient driven TE dominated transport, respectively. ${ }^{9,28}$ However, to be fully consistent with the observations an assumption of a sub-dominant electron temperature gradient driven TE mode was necessary. Further experimental studies in JET plasmas ${ }^{26}$ showed a favorable impact of ICRH in preventing the accumulation of metallic 
impurities in the core. However, in this case a theoretical explanation based on the presence of a TE mode driving outward impurity flux would be unsatisfactory since these plasmas were ITG dominated.

Recently, a new possibility has emerged from the work reported in Refs. 29-31 where the observed outward directed impurity flux is explained as an effect of poloidal asymmetries generated by the ICRH. The temperature anisotropy due to ICRH will trap the minority heated ions on the low field side, leading to the establishment of a poloidally varying "equilibrium" (i.e., non-fluctuating) electrostatic potential (such asymmetries have experimentally been demonstrated in Ref. 32). The associated $\mathbf{E} \times \mathbf{B}$ drift acting as another degree of freedom for impurities to respond to electrostatic perturbations modifies the fluctuating impurity distribution. It has been shown that under experimentally relevant conditions the contribution of these $\mathbf{E} \times \mathbf{B}$ drifts to the impurity particle transport can be outward and might dominate the resulting steady state impurity density gradient even in ITG dominant regimes.

The density peaking of high- $Z$ impurities in density or temperature gradient driven TE dominated plasmas under RF induced poloidal asymmetries is yet to be analyzed; this is the aim of the present paper. Apart from numerical simulations with GYRO ${ }^{33}$ (mainly linear simulations, but a few nonlinear simulations are also performed for comparison) an analytical model including the effect of poloidal asymmetries $^{31,34}$ is utilized. The model is based on a solution to the linearized gyrokinetic equation, and it suggests that the impurity velocity pinch is governed by three separate contributions: one related to the magnetic drifts (combined effects of curvature and thermodiffusion pinch), another to the parallel impurity velocity pinch, and a third part arising due the $\mathbf{E} \times \mathbf{B}$ drift in a poloidally varying equilibrium electrostatic potential. Using this model, we present a systematic comparison of impurity transport driven by density and temperature driven trapped electron modes, highlighting the effect of the parallel impurity motion, collisions, magnetic geometry (shear and safety factor), and poloidal asymmetries.

The remainder of the paper is organized as follows. In Sec. II, we describe the baseline density and temperature gradient driven TE mode cases and the linear stability characteristics of them. In Sec. III, the density peaking of high- $Z$ trace impurities is analyzed, and the dependence on relevant plasma parameters, such as electron density and temperature gradients, ion-to-electron temperature ratio, safety factor, and magnetic shear, is presented. Impurity peaking factors are calculated in cases where the impurities are poloidally symmetrically distributed but also in cases where a poloidally varying potential is present. The results are discussed and summarized in Sec. IV.

\section{STABILITY}

The TE mode instability is driven by the electron logarithmic temperature gradient, $a / L_{T e}$, and/or the logarithmic density gradients, $a / L_{n}$, whereas ITG modes are driven by the ion logarithmic temperature gradient $a / L_{T i}$. Here $L_{n \alpha}$ $=-\left[\partial\left(\ln n_{\alpha}\right) / \partial r\right]^{-1}$ and $L_{T \alpha}=-\left[\partial\left(\ln T_{\alpha}\right) / \partial r\right]^{-1}$ represent the density and temperature scale lengths of particle species $\alpha$, respectively, and $a$ the outermost minor radius of the plasma.

In this paper we will study two baseline collisionless TE mode cases: one driven by the density gradients and one driven by the electron temperature gradient. For the second case the ion temperature gradient is set to zero, in order to obtain pure TE turbulence. This represents a situation with dominant central electron heating. Our baseline cases have the following local profile and magnetic geometry parameters:

Case I: Density gradient driven TE mode

$$
\begin{aligned}
& R_{0} / a=3, r_{0} / a=0.5, q=2, s=1, \\
& \beta=0, a / L_{n}=3, a / L_{T e}=a / L_{T i}=a / L_{T z}=1, \\
& T_{e}=T_{i}=T_{z}, \hat{\nu}_{e i}=0, \rho_{s 0} / a=0.0035 .
\end{aligned}
$$

Case II: Electron temperature gradient driven TE mode

$$
\begin{aligned}
& R_{0} / a=3, r_{0} / a=0.375, q=1.4, s=0.8, \beta=0, \\
& \quad a / L_{n}=1, a / L_{T e}=7 / 3, a / L_{T z}=7 / 3, \\
& \quad a / L_{T i}=0, T_{e}=T_{i}=T_{z}, \hat{\nu}_{e i}=0, \rho_{s 0} / a=0.0035 .
\end{aligned}
$$

Here the indices represent electrons (e), main ions $(i)$, and impurities $(z)$. The density gradient driven case (Case I) is one of the GYRO standard cases in the GYRO nonlinear gyrokinetic simulation database. ${ }^{35}$ The electron temperature gradient driven case (Case II) have been used in the fluid simulations presented in Ref. 11. In both cases fully ionized nickel, $Z=28$, is introduced in trace (i.e., $Z n_{z} / n_{e} \ll 1$ ) quantities $n_{z} / n_{e}=2 \times 10^{-3}$; however, note that $Z^{2} n_{z} / n_{e} \sim \mathcal{O}(1)$ which is important for the approximate model of the impurity peaking factor we will use. The use of nickel will ease comparison with previous work, e.g., Refs. 9 and 31, but the main conclusions will be valid for any high- $Z$ impurity. $R_{0}$ is the major radius of the magnetic axis and $r_{0}$ the local reference minor radius, $q$ is the safety factor, and $s=(r / q)(d q / d r)$ the magnetic shear, while $\beta$ represents the ratio of plasma pressure to magnetic pressure. We note that electromagnetic fluctuations appearing for finite $\beta$ have negligible effect on TE modes as trapped electrons cannot carry parallel current. In the main part of the paper, the plasma is assumed to be hot enough for collisions to be ignored and consequently the electron-ion collision frequency is $\hat{\nu}_{e i}=0$, except when it is stated otherwise.

This paper considers turbulent fluxes. Neoclassical simulations of the baseline cases with $\mathrm{NEO}^{36}$ using $\hat{\nu}_{e i}=0.0058 c_{s} / a$ (corresponding to $T_{e}=7 \mathrm{keV}, n_{i}=3 \times 10^{19} \mathrm{~m}^{-3}, \ln \Lambda=17$, and $a=1 \mathrm{~m}$ ) result in fluxes that are an order of magnitude smaller than the turbulent fluxes from nonlinear GYRO simulations.

Nonlinear GYRO simulations of the baseline cases show that the largest fluxes occur in the vicinity of $k_{\theta} \rho_{s}=0.15$ for both of them; see Fig. 1 showing the poloidal wave number spectra of the gyro-Bohm normalized electron energy fluxes. Here $k_{\theta}$ is the poloidal wave-number and $\rho_{s}$ $=\rho_{s 0}(1+\epsilon \cos \theta)$ the ion sound Larmor radius, where $\rho_{s 0}$ denotes $\rho_{s}$ at $R_{0}, \epsilon=r_{0} / R_{0}$ is the inverse aspect ratio, and $\theta$ the extended poloidal angle. Consequently $k_{\theta} \rho_{s}=0.15$ is 
(Total,elec,energy)

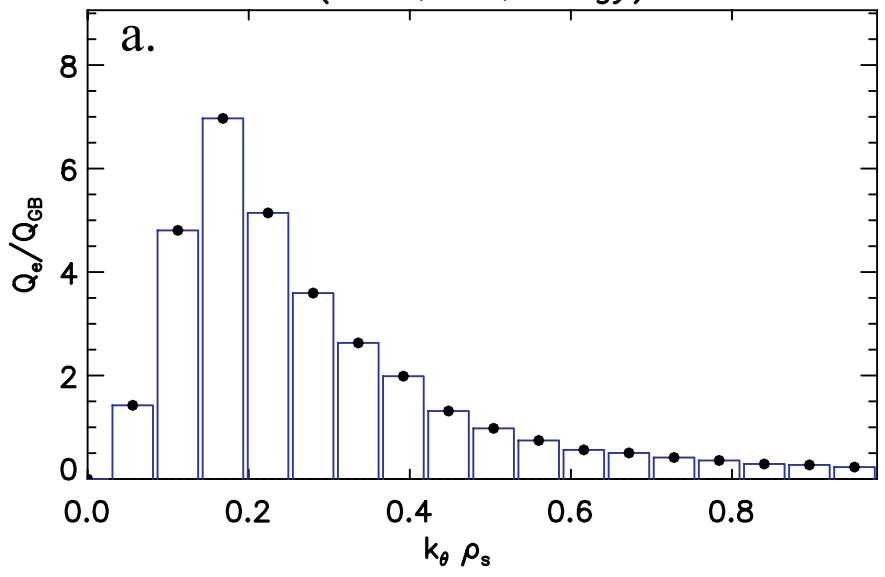

(Total,elec,energy)

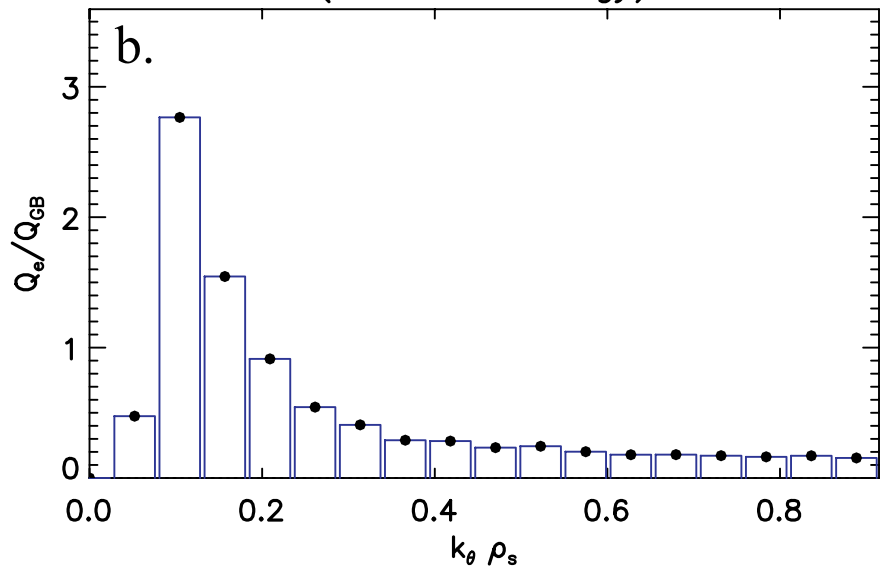

FIG. 1. Normalized electron energy fluxes $Q_{e} / Q_{\mathrm{GB}}$ as functions of poloidal wave-number $k_{\theta} \rho_{s}$ from nonlinear GYRo simulations for Case I (a) and Case II (b).

used in the quasilinear simulations for both cases. Note, however, that the maximum of the linear growth rates $\gamma$ are located at higher $k_{\theta} \rho_{s}$ as shown in Fig. 2. As expected for a temperature gradient driven TE mode, the frequency increases, i.e., it propagates faster in the electron diamagnetic direction, with increasing $k_{\theta}$ in Case II. ${ }^{9}$ Frequencies are given in $c_{s} / a$ units, where $c_{s}=\left(T_{e} / m_{i}\right)^{1 / 2}$ is the ion sound speed and $r_{0}=a$ for the last closed flux surface.

The perturbed electrostatic potential $\phi$ and eigenvalues $\omega=\omega_{r}+i \gamma$ are obtained by linear electrostatic gyrokinetic initial-value calculations with GYRO. ${ }^{33}$ Linear initial-value studies only consider the most unstable mode, and any subdominant modes are neglected. In the simulations a model Grad-Shafranov magnetic equilibrium was used, where the $\mathcal{O}(\epsilon)$ corrections to the drift frequencies are retained. Fluxtube (periodic) boundary conditions were used, with a 128 point velocity space grid ( 8 energies, 8 pitch angles, and two signs of velocity), the number of radial grid points is 6 , and the number of poloidal grid points along particle orbits is 20 for trapped particles. The location of the highest energy grid point is at $m_{i} v^{2} /\left(2 T_{i}\right)=6$. The ions were taken to be gyrokinetic and the electrons to be drift kinetic with the mass ratio $\left(m_{i} / m_{e}\right)^{1 / 2}=60$.

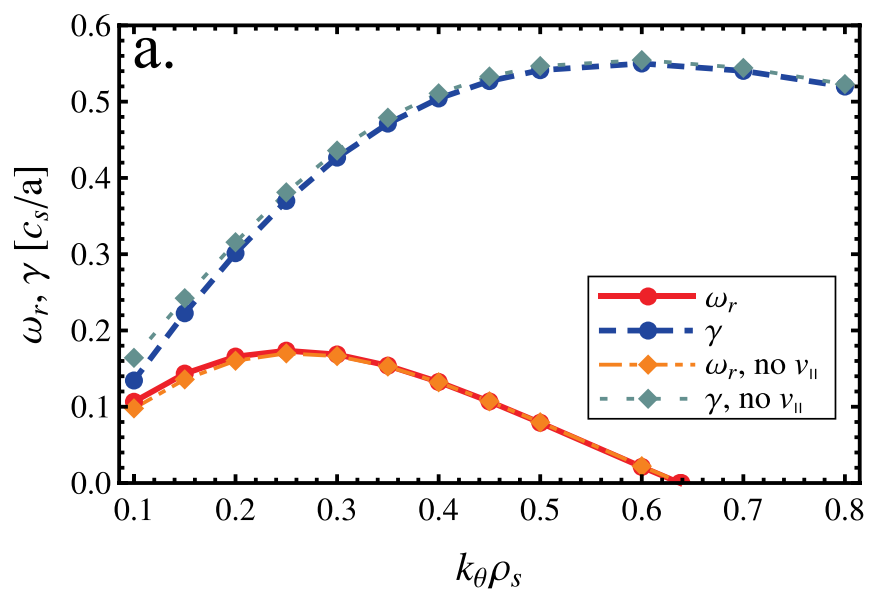

The nonlinear electrostatic GYRO simulations performed for the baseline cases also use gyrokinetic ions and drift kinetic electrons and the same velocity resolution as the linear simulations. At least 18 toroidal modes are used to model $1 / 4$ th of the torus, with the highest resolved poloidal wave number being $k_{\theta} \rho_{s} \approx 0.9$. The number of radial grid points is 200. The simulations are run with the integration time step $\Delta t=0.01 a / c_{s}$ for $t>200 a / c_{s}$.

Introducing a small collision rate is expected to have stabilizing effect on the collisionless TE mode because the trapped electrons, driving the instability, can be detrapped. One of the most interesting distinctions between the two different branches of the TE modes we study concerns the dependence of the linear growth rate on collisionality. If the TE mode is mainly driven by the electron temperature gradient, the mode is completely stabilized by collisions at a very low collision frequency, as was pointed out in Ref. 37. As shown in Fig. 3, this was verified also in our simulations, where Case II was suppressed already for $\hat{\nu}_{e i}>0.015 c_{s} / a$ while Case I persisted even for very high collisionalities, also consistent with earlier studies of density gradient driven TE modes ${ }^{1,37}$ (note that the $\hat{\nu}_{e i}$-ranges plotted are different, and that $\omega_{r}$ is positive for modes propagating to the electron

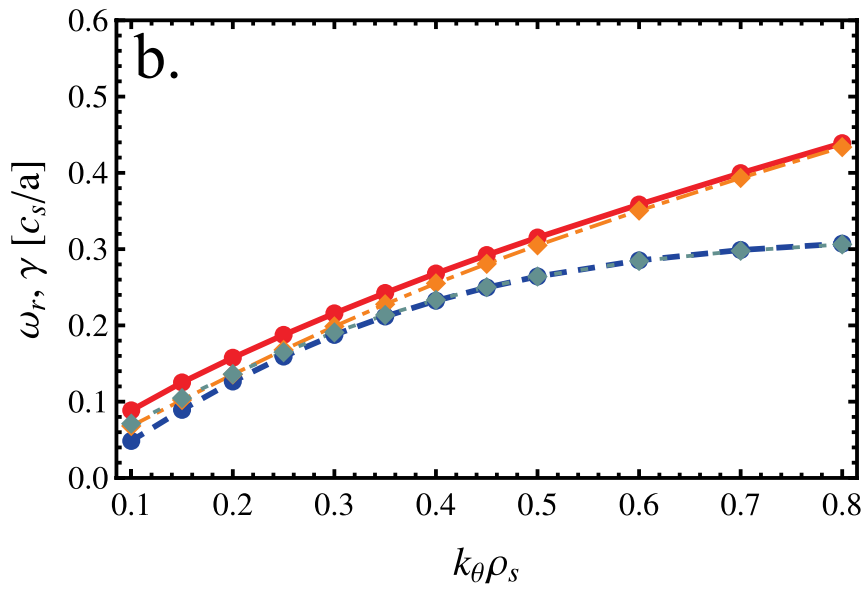

FIG. 2. Linear growth rate $\gamma$ (circle markers, blue dashed lines) and real mode frequency $\omega_{r}$ (circle markers, red solid lines) as functions of poloidal wavenumber $k_{\theta} \rho_{s}$ for Case I (a) and Case II (b). Linear growth rate $\gamma$ (diamond markers, green dotted lines) and real mode frequency $\omega_{r}$ (diamond markers, orange dash-dotted lines) for the same cases but with parallel ion motion neglected in GYRO. 

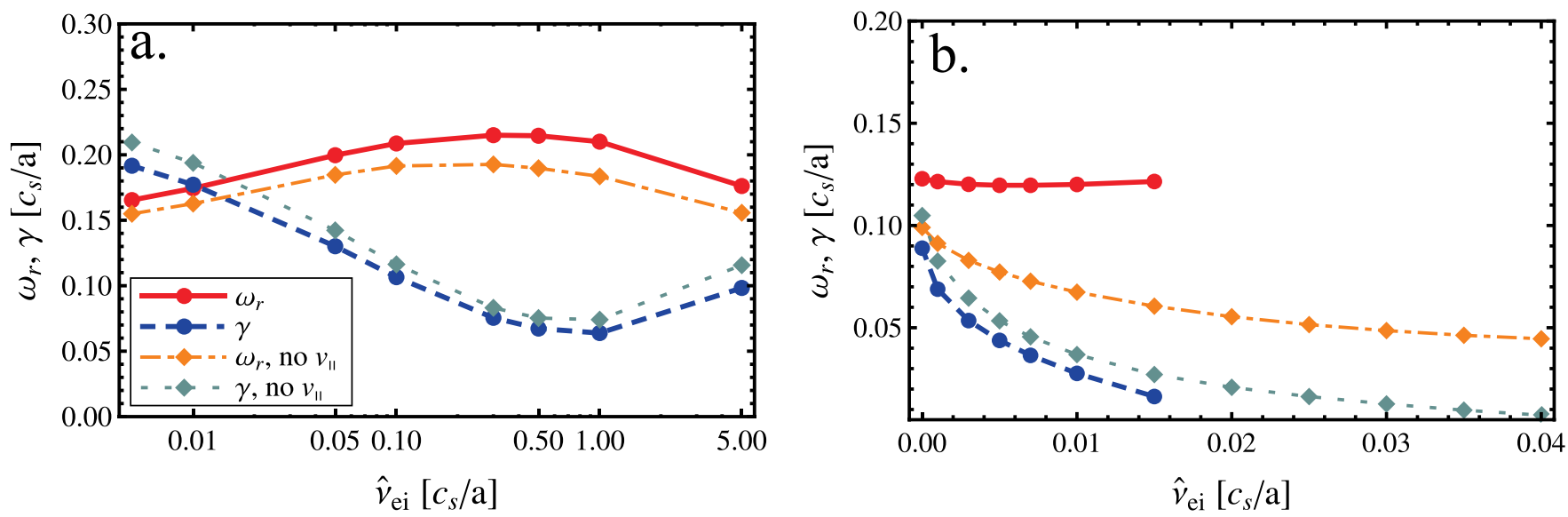

FIG. 3. Linear growth rate $\gamma$ (circle markers, blue dashed lines) and real mode frequency $\omega_{r}$ (circle markers, red solid lines) as functions of electron-ion collision frequency $\hat{\nu}_{e i}$ for Case I (a) (note the logarithmic $\hat{\nu}_{e i}$-axis) and Case II (b). Linear growth rate $\gamma$ (diamond markers, green dotted lines) and real mode frequency $\omega_{r}$ (diamond markers, orange dash-dotted lines) for the same cases but with parallel ion motion neglected in GYRO.

diamagnetic direction according to GYRO conventions). For $\hat{\nu}_{e i}>1.0 c_{s} / a$ Case I even exhibits an increase in growth rate with increasing collisionality. This could indicate that the mode is turning into a dissipative TE mode, but it can also suggest that TE modes driven by density gradients remain unstable even at large collisionalities. Further it can be noted that neglecting parallel ion motion in the simulations leads to a small to moderate reduction of the real mode frequency while the growth rate is almost unaffected.

Figure 4 shows how the perturbed potential varies with the extended poloidal angle. We see that both cases exhibit highly ballooned structures, concentrated to $\theta \in[-\pi, \pi]$, and that there is no significant difference between including and not including parallel ion motion (note that the effect of parallel ion motion is expected to be stronger in cases of lower $k_{\theta} \rho_{s}$ ).

\section{IMPURITY DENSITY PEAKING}

In this section, the zero flux density gradient (peaking factor) for trace impurities is analyzed. We utilize a semianalytical model introduced in Ref. 34, where the effect of a poloidally varying equilibrium electrostatic potential $\phi_{E}$ is included. The focus is on the poloidally varying part of the

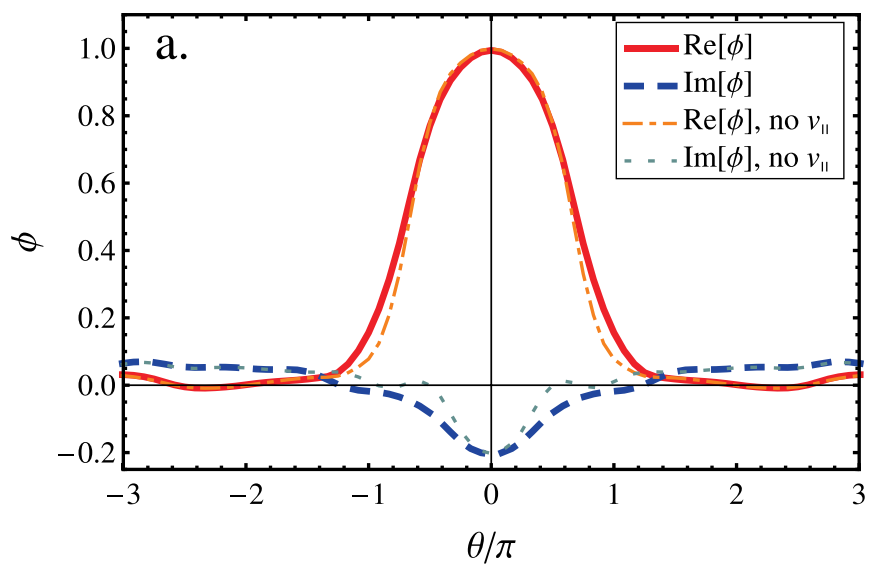

electrostatic potential, and effects caused by a radial electric field, such as toroidal rotation, are neglected. However, we note that the Coriolis drift or the poloidal redistribution of impurities due to centrifugal forces can have a nonnegligible influence on impurity transport, as found in recent works. ${ }^{38-41}$ The poloidally varying potential introduces an $\mathbf{E} \times \mathbf{B}$ drift frequency labeled $\omega_{E}$ in the GK equation (3), which disappears, $\omega_{E}=0$, in the symmetric case (note that GYRO only considers the poloidally symmetric case). Poloidal variation can be caused by the presence of a species with strong temperature anisotropy, ${ }^{32}$ which is the case in discharges with radio frequency (RF) heating of minority ions on the outboard side. ${ }^{42,43}$

The poloidally varying potential is assumed to be weak in the sense that $e \Delta \phi_{E} / T_{\alpha} \ll 1$, where $T_{\alpha}$ is the temperature of species $\alpha$. This implies that the effect of poloidal asymmetries on the main species can be neglected. This justifies the use of GYRO simulations neglecting poloidal asymmetries to obtain linear mode characteristics. By requiring $Z \gg 1$, we allow $Z e \Delta \phi_{E} / T_{z} \sim \mathcal{O}(1)$, and consequently the impurities can be poloidally asymmetrically distributed. Hence their $\mathbf{E} \times \mathbf{B}$ drift in the poloidally varying electrostatic potential $\phi_{E}$ is not negligible. This model was presented in Ref. 31,

FIG. 4. Linear parallel mode structure of the perturbed potential $\phi(\theta)$ for Case I (a) and Case II (b). Real part (red solid lines) and imaginary part (blue dashed lines) of $\phi$. Real part (orange dash-dotted lines) and imaginary part (green dotted lines) of $\phi$ for the same cases but with parallel ion motion neglected in GYRO. Note that the actual resolution of the simulation covers $\theta / \pi=(-7,5)$, by GYRO convention. 
and we refer to this work for more details. The model we use for the equilibrium electrostatic potential is given by Eq. (11) in Ref. 31

$$
Z e \phi_{E} / T_{z}=-\kappa \cos (\theta-\delta)
$$

where $\delta$ represents the angular position where the impurity density has its maximum and $\kappa$ sets the strength of the poloidal asymmetry. Thus the impurity density will be assumed to vary according to $n_{z}(\theta, r)=n_{z 0}(r) \mathcal{N}(\theta)$ with $\mathcal{N}(\theta)$ $=\exp [\kappa \cos (\theta-\delta)]$. In the model for ion cyclotron resonance heating (ICRH) driven asymmetries presented in Ref. $43 \delta=\pi$ is obtained; however, since impurity accumulation has also been observed at other poloidal locations we shall consider $\delta=0$ and $\delta=\pi / 2$ cases as well.

Note that in contrast to Ref. 31, the work presented here as well as in Ref. 34 retains the effects of the parallel ion streaming in the GK equation (3). In the case of transport driven by TE mode turbulence, as we will show here, this term can significantly affect the impurity peaking.

\section{A. Zero flux impurity density gradient}

We consider particle transport driven by a single, representative, toroidal mode. The impurity peaking factor is calculated by requiring the linear impurity flux $\Gamma_{z}$ to vanish

$0=\left\langle\Gamma_{z}\right\rangle \equiv\left\langle\Im\left[-\frac{k_{\theta}}{B} \hat{n}_{z} \phi^{*}\right]\right\rangle=\left\langle\Im\left[-\frac{k_{\theta}}{B} \int d^{3} v J_{0}\left(z_{z}\right) g_{z} \phi^{*}\right]\right\rangle$,

where $\langle\cdot\rangle$ denotes the flux surface average, $\Im[\cdot]$ denotes imaginary part, $\hat{n}_{z}$ is the perturbed impurity density, $g_{z}$ the non-adiabatic part of the perturbed impurity distribution function, $J_{0}$ is the Bessel function of the first kind, $z_{z}=$ $k_{\perp} v_{\perp} / \omega_{c z}, \omega_{c z}=Z e B / m_{z}$ is the cyclotron frequency, and $k_{\perp}=\left(1+s^{2} \theta^{2}\right)^{1 / 2} k_{\theta}$. Furthermore $m_{z}$ is the impurity mass, $\phi^{*}$ is the complex conjugate of the perturbed electrostatic potential, and $B$ is the strength of the equilibrium magnetic field. The subscripts $\|$ and ${ }_{\perp}$ denote the parallel and perpendicular directions with respect to the magnetic field.

The non-adiabatic perturbed impurity distribution $g_{z}$ is obtained from the linearized GK equation

$$
\begin{gathered}
\left.\frac{v_{\|}}{q R} \frac{\partial g_{z}}{\partial \theta}\right|_{\mathcal{E}, \mu}-i\left(\omega-\omega_{D z}-\omega_{E}\right) g_{z}-C\left[g_{z}\right] \\
=-i \frac{Z e f_{z 0}}{T_{z}}\left(\omega-\omega_{* z}^{T}\right) \phi J_{0}\left(z_{z}\right),
\end{gathered}
$$

where $\omega=\omega_{r}+i \gamma$ is the mode frequency, $f_{z 0}=n_{z 0}\left(m_{z}\right.$ $\left./ 2 \pi T_{z}\right)^{3 / 2} \exp \left(-\mathcal{E} / T_{z}\right)$ is the equilibrium Maxwellian distribution function, $\mathcal{E}=m_{z} v^{2} / 2+Z e \phi_{E}$ is the total unperturbed energy, $\mu=m_{z} v_{\perp}^{2} /(2 B)$ is the magnetic moment, $n_{z}(\mathbf{r})=$ $n_{z 0} \exp \left[-Z e \phi_{E}(\mathbf{r}) / T_{z}\right]$ is the poloidally varying impurity density, and $n_{z 0}$ is a flux function. The diamagnetic frequency is defined as $\omega_{* z}=-k_{\theta} T_{z} / Z e B L_{n z}$ and $\omega_{* z}^{T}=\omega_{* z}\left[1+\left(x^{2}\right.\right.$ $\left.-3 / 2) L_{n z} / L_{T z}\right]$, and $x=v / v_{T z}$ represents velocity normalized to the thermal speed $v_{T z}=\left(2 T_{z} / m_{z}\right)^{1 / 2}$. The magnetic drift frequency is $\omega_{D z}=-2 k_{\theta} T_{z}\left(x_{\perp}^{2} / 2+x_{\|}^{2}\right) \mathcal{D}(\theta) /\left(m_{z} \omega_{c z} R\right)$, where $\mathcal{D}(\theta)=\cos \theta+s \theta \sin \theta$. The $\mathbf{E} \times \mathbf{B}$ drift frequency of the particles in the equilibrium electrostatic field $\omega_{E}$ is

$$
\omega_{E}=-\frac{k_{\theta}}{B} \frac{s}{r} \frac{\partial \phi_{E}}{\partial \theta}
$$

and was derived in Appendix A of Ref. 31 (here, the $\partial \phi_{E} / \partial r$ part is dropped). $C[\cdot]$ is the collision operator.

A solution to Eq. (3), the subsequent expression for the peaking factor, is presented in Ref. 34. It is a perturbative solution in the small parameter $Z^{-1 / 2} \ll 1$, keeping terms up to $\mathcal{O}\left(Z^{-1}\right)$ in the expansion of $g_{z}$. This is based on the fact that $\omega_{D z} / \omega, \omega_{* z}^{T} / \omega$ and $J_{0}\left(z_{z}\right)-1 \approx-z_{z}^{2} / 4$ are all $\sim 1 / Z$ small and that our ordering $Z e \phi_{E} / T_{z} \sim \mathcal{O}(1)$ requires that $\omega_{E} / \omega$ also is formally $\sim 1 / Z$ small. The solution assumes that impurity self-collisions dominate over collisions with unlike species, which follows from the ordering $n_{z} Z^{2} / n_{e} \sim \mathcal{O}(1)$, and the self-collisions are modeled by the full linearized impurity-impurity collision operator $C_{z z}^{(l)}$, maintaining the conservation properties and self-adjointness. As earlier mentioned it is also assumed that $\phi$ and $\omega$ are known from the solution of the linear gyrokinetic-Maxwell system (obtained from GYRO) and that they are unaffected by the presence of trace impurities and, in particular, their poloidal asymmetry. The impurity transit frequency $v_{\|} /(q R)$ is typically much smaller than the mode frequency $\omega$, and therefore magnetic (and electrostatic) trapping of the impurities can be neglected.

The expression for the impurity peaking factor, $a / L_{n z}^{0}$, is given in Eq. (8) of Ref. 34 and can be modified into

$$
\begin{aligned}
\frac{a}{L_{n z}^{0}}= & 2 \frac{a}{R_{0}}\langle\mathcal{D}\rangle_{\phi}+\frac{a}{r} s \kappa\langle\theta \sin (\theta-\delta)\rangle_{\phi} \\
& -\frac{2 a^{2}}{\left(q R_{0}\right)^{2} k_{\theta} \rho_{s 0}} \frac{Z m_{i}}{m_{z}} \frac{c_{s}}{a} \frac{\omega_{r}}{\omega_{r}^{2}+\gamma^{2}}\left\langle\left|\frac{\partial \phi}{\partial \theta}\right|^{2} /|\phi|^{2}\right\rangle_{\phi},
\end{aligned}
$$

where $\langle\ldots\rangle_{\phi}=\left\langle\ldots \mathcal{N}|\phi|^{2}\right\rangle /\left\langle\mathcal{N}|\phi|^{2}\right\rangle$. To find this expression $\mathcal{O}(\epsilon)$ corrections together with finite values of the mode eigenfunction outside the range $[-\pi, \pi]$ of the extended poloidal angle have been neglected. As a consequence the expression is not valid in cases of highly elongated ballooning eigenfunctions, but as shown in Fig. 4 the TE modes we study have a $\phi$ localized to this interval. It is interesting to note that up to the considered order, $\mathcal{O}\left(Z^{-1}\right)$, both finite Larmor radius (FLR) effects and the effects of collisions do not appear. Furthermore we see that $a / L_{n z}^{0}$ consists of three terms: the first term of Eq. (5) represents the contribution of the magnetic drift, the second term stems from the $\mathbf{E} \times \mathbf{B}$ drift and is only non-zero when there is a poloidally varying potential, and the last term arises because of the impurity parallel dynamics. The first two terms were present already in Eq. (14) of Ref. 31, but in that expression parallel ion/impurity dynamics was neglected. The term due to parallel dynamics contains only non-negative quantities, except $\omega_{r}$, and consequently impurity parallel dynamics acts to increase (decrease) the impurity peaking if $\omega_{r}$ is negative (positive). This leads to the conclusion that when the TE mode is the dominant instability $\left(\omega_{r}>0\right)$ the parallel dynamics should 
act to decrease the impurity peaking, while the opposite is true for ITG modes. We note that in the poloidally symmetric $(\mathcal{N}=1)$ case the parallel compressibility term in Eq. (5) is consistent with Eqs. (9) and (10) of Ref. 7 in the high $Z$ limit when $k_{\|}^{2}$ is defined as $\left(q R_{0}\right)^{-2}\left\langle\left|\partial_{\theta} \phi\right|^{2}\right\rangle /\left\langle|\phi|^{2}\right\rangle$. The effect of parallel dynamics was pointed out already in Ref. 7 , where it was observed that in conditions of strong electron temperature gradient the mechanism can be large enough to reverse the total pinch of trace impurities from inwards to outwards.

\section{B. Parametric dependences in poloidally symmetric cases}

In the present section we show how the impurity peaking factor and mode eigenvalues depend on electron temperature gradient, ion-to-electron temperature ratio, electron density gradient, and safety factor, when $\phi_{E}$ is poloidally symmetric. Results are presented for both Case I and Case II, when parallel ion/impurity dynamics are included as well as when they are neglected. Results from Eq. (5) are compared to simulations by GYRO. Although the analytical model for the peaking factor given in Eq. (5) is based on a single linear mode, for completeness we will present a few cases where peaking factors have been determined from nonlinear simulations with GYRO. The reason for this is to provide further insight into the validity of the analytical model. Note that the analytical results from Eq. (5) are not expected to agree exactly even with the linear results of GYRO since the approximation only retains effects up to order $1 / Z$ as well as neglects $\mathcal{O}(\epsilon)$ corrections. Note that for cases with parallel ion/impurity dynamics neglected, the mode characteristics come from GYRO simulations where parallel compressibility effects have been turned off. Therefore they differ slightly from the magnetic drift contribution in cases where parallel dynamics is included. This can be observed in, e.g., Figs. 5(a) and 5(b), comparing the blue dotted line with the orange dashed line.

\section{Temperature and temperature gradient dependences}

Figures 5(a) and 5(b) show how the nickel peaking factor varies with electron temperature gradient and Figs. 5(c) and $5(\mathrm{~d})$ the corresponding eigenvalues. For both cases there is a slight increase in peaking for increasing $a / L_{T e}$, but the dependence is generally very weak. This is what is expected from Eq. (5), where there is no explicit dependence on $a / L_{T e}$. Instead the variations are caused by changes in mode frequency, shown in Figs. 5(c) and 5(d), and perturbed potential through the parallel compressibility term, which is reflected in the fact that almost no variation at all is observed
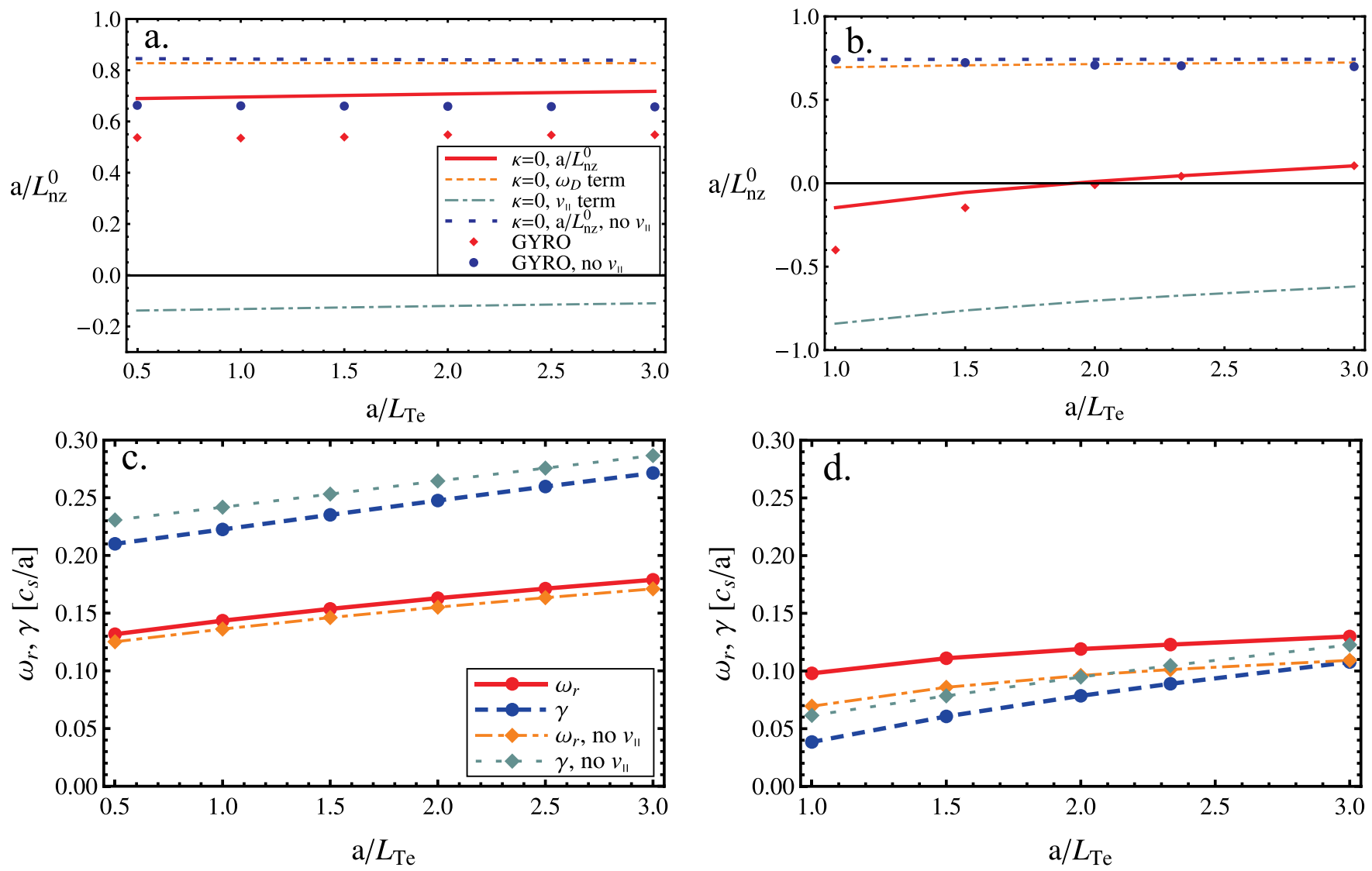

FIG. 5. (a), (b) Impurity peaking factor for trace nickel as function of electron temperature gradient $a / L_{T e}$ for Case I (a) and Case II (b). Red solid line is the peaking factor from Eq. (5), orange dashed line the magnetic drifts contribution, and green dashed-dotted line the parallel compressibility contribution. Blue dotted line is the peaking factor from Eq. (5) without parallel compressibility effects. Red diamonds and blue dots correspond to GYRO results with and without parallel compressibility effects, respectively. (c),(d) Linear growth rate $\gamma$ (circle markers, blue dashed lines) and real mode frequency $\omega_{r}$ (circle markers, red solid lines) as functions of electron temperature gradient $a / L_{T e}$ for Case I (c) and Case II (d). Linear growth rate $\gamma$ (diamond markers, green dotted lines) and real mode frequency $\omega_{r}$ (diamond markers, orange dash-dotted lines) for the same cases but with parallel ion motion neglected in GYRO. 

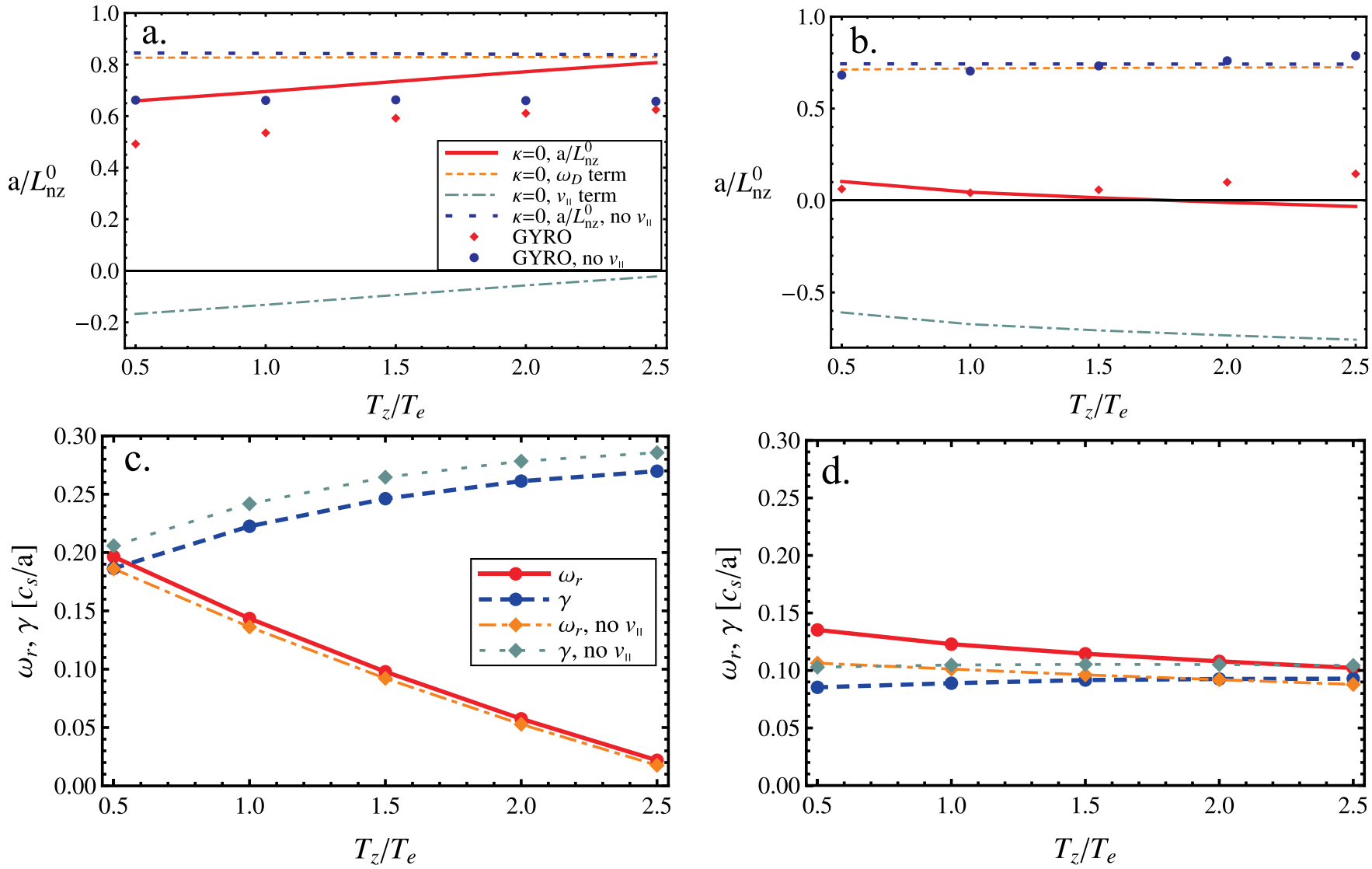

FIG. 6. (a), (b) Impurity peaking factor for trace nickel as function of ion-to-electron temperature ratio $T_{i} / T_{e}$ (note that $T_{i} / T_{e}=T_{z} / T_{e}$ ) for Case I (a) and Case II (b). Red solid line is the peaking factor from Eq. (5), orange dashed line the magnetic drifts contribution, and green dashed-dotted line the parallel compressibility contribution. Blue dotted line is the peaking factor from Eq. (5) without parallel compressibility effects. Red diamonds and blue dots correspond to GYRO results with and without parallel compressibility effects, respectively. (c),(d) Linear growth rate $\gamma$ (circle markers, blue dashed lines) and real mode frequency $\omega_{r}$ (circle markers, red solid lines) as functions of ion-to-electron temperature ratio $T_{i} / T_{e}$ for Case I (c) and Case II (d). Linear growth rate $\gamma$ (diamond markers, green dotted lines) and real mode frequency $\omega_{r}$ (diamond markers, orange dash-dotted lines) for the same cases but with parallel ion motion neglected in GYRO.

for the results with parallel compressibility neglected. Both the linear growth rate and real mode frequency increase with electron temperature gradient, where the increase in growth rate is expected since electron temperature gradient is one of the drives for TE modes.

The peaking factor dependence on ion-to-electron temperature ratio (note that $T_{i} / T_{e}=T_{z} / T_{e}$ ) is illustrated in Figs. 6(a) and 6(b). We see that it exhibits a similar dependence as to $a / L_{T e}$, which is weak and only enters through changes in $\omega_{r}, \gamma$, and $\phi$. How $\omega_{r}$ and $\gamma$ are affected by changes in $T_{i} / T_{e}$ is shown in Figs. 6(c) and 6(d). Case I shows a significant increase in growth rate with $T_{i} / T_{e}$ and decrease in real mode frequency, while Case II is almost unaffected. This implies that as $T_{i} / T_{e}$ increases, Case I is approaching an ITG mode. Similar trends were reported in terms of mode frequencies and growth rates in Ref. 13 where temperature and density gradient driven TE mode dominated plasmas were compared (having Ohmic and electron cyclotron heating, respectively); in the density gradient driven case, $\omega_{r}(\gamma)$ was found to decrease (increase) with increasing $T_{i} / T_{e}$, while weaker temperature ratio dependences of $\omega_{r}$ and $\gamma$ were observed in the temperature gradient driven case.

Earlier studies of TE modes have shown that the linear growth rate is expected to decrease with increasing $T_{i} / T_{e}$, as seen in Fig. 5 of Ref. 44, which is seemingly in disagreement with what is observed here. However, it has to be noted that the simulation in Ref. 44 was performed keeping $k_{\theta} \rho_{i}$ fixed, while here $k_{\theta} \rho_{s}$ is fixed. Since $k_{\theta} \rho_{s} \sim\left(T_{e} / T_{i}\right)^{1 / 2} k_{\theta} \rho_{i}$, a parametric scan over $T_{i} / T_{e}$ keeping $k_{\theta} \rho_{i}$ fixed results in varying $k_{\theta} \rho_{s}$ accordingly, and thus these scalings are not comparable. Furthermore in Ref. 44 results are presented with $\gamma$ normalized to $v_{T i} / L_{n} \propto \sqrt{T_{i}}$, while here we normalize to $c_{s} / a \propto \sqrt{T_{e}}$. A test was performed, varying $k_{\theta} \rho_{s}$ to keep $k_{\theta} \rho_{i}$ fixed, and a similar decrease with increasing $T_{i} / T_{e}$ was found. Also Ref. 45 finds an increase in linear growth rate with increasing $T_{i} / T_{e}$ in agreement with our observations.

A similar rather weak dependence of the impurity peaking on $a / L_{T e}$ and $T_{i} / T_{e}$ was found in gyrokinetic simulations by Ref. 9 as long as the most unstable mode remained the same. As observed in Case I here, for density gradient driven TE modes the peaking factor is typically positive. For temperature gradient driven TE modes, however, it can be negative which is also found in Case II where it is close to zero or even below, as seen in Figs. 5(b) and 6(b).

There is a small discrepancy between the values found by GYRO and the values calculated from Eq. (5). For Case I, Eq. (5) systematically overestimates the magnetic drift contribution, while for Case II discrepancies arise mainly due to 
the parallel compressibility term. Still we see that the effect of the parallel impurity dynamics is rather well explained by the approximate solution.

From Figs. 5(c), 5(d), 6(c), and 6(d) we see that neglecting the parallel ion motion in the GK equation $\left(v_{\|} /(q R) \partial g_{z} / \partial \theta=0\right.$ in Eq. (3)) only has a minor impact on the TE mode frequencies.

\section{Density gradient dependence}

The scaling of the impurity density peaking with the main species density peaking is shown in Figs. 7(a) and 7(b), and the corresponding eigenvalues in Figs. 7(c) and 7(d). It is interesting to note how the impurity peaking shows a significant decrease in Case I for $a / L_{n e}=1.0$, compared to the other values, which is almost solely due to the change in the factor $\omega_{r} /\left(\omega_{r}^{2}+\gamma^{2}\right)$ in the parallel compressibility term of Eq. (5) (the variation in $\phi(\theta)$ with $a / L_{n e}$ is rather weak). The change in magnitude of this factor is clearly observed in Fig. $7(\mathrm{c})$, where for $a / L_{n e}=1.0, \omega_{r}$ and $\gamma$ are comparable in size while for the other points $\gamma$ is significantly larger. For density gradient driven TE modes a strong reduction in the peaking factor towards weaker density gradients has previously been reported in Ref. 46, using both quasilinear and nonlinear GENE $^{47}$ simulations. In Case II $\omega_{r} /\left(\omega_{r}^{2}+\gamma^{2}\right)$ experience a small reduction with increasing $a / L_{n e}$, and this is reflected in the impurity peaking factor which is increased because of the smaller size of the parallel compressibility term. For $a / L_{n e}=0.5$ this case has a negative peaking factor.

For $a / L_{n e}=1.0$ we could expect that Case I changes to become a temperature gradient driven TE mode; since then the density and temperature gradients are comparable $\left(a / L_{T e}=1.0\right.$ for Case I). It seems that for temperature gradient driven TE modes the magnitude of the ratio of $\omega_{r}$ compared to $\gamma$ is usually larger than for density gradient driven TE modes. This has the consequence that the parallel dynamics becomes more important in reducing the impurity density peaking for temperature gradient driven TE modes. Further, we note that as could be expected from Eq. (5) the peaking factors are unaffected by changes in $a / L_{n e}$ if parallel compressibility is neglected. For both cases the linear growth rate and real mode frequency increase with increasing $a / L_{n e}$. The eigenvalues do not change much by neglecting parallel compressibility, except in Case I with $a / L_{n e}=1.0$ where an ITG mode is found instead of a TE mode.

\section{Safety factor dependence}

From the last term of Eq. (5), it is expected that the influence of impurity parallel dynamics on the impurity peaking factor is strongly reduced with increasing safety factor. This is also what is observed in simulations, where the
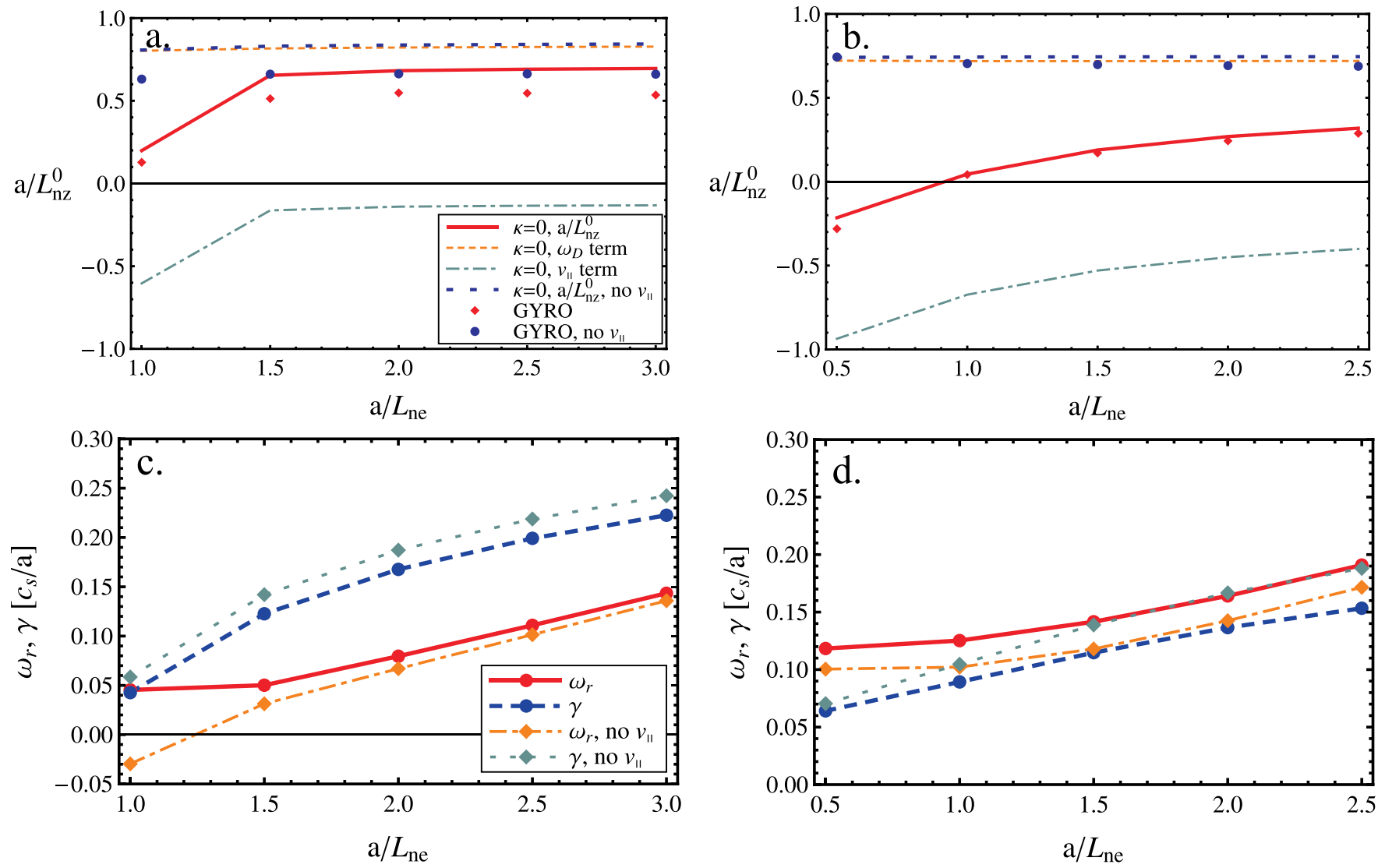

FIG. 7. (a), (b) Impurity peaking factor for trace nickel as function of electron density gradient $a / L_{n e}$ for Case I (a) and Case II (b). Red solid line is the peaking factor from Eq. (5), orange dashed line the magnetic drifts contribution, and green dashed-dotted line the parallel compressibility contribution. Blue dotted line is the peaking factor from Eq. (5) without parallel compressibility effects. Red diamonds and blue dots correspond to GYRO results with and without parallel compressibility effects, respectively. (c),(d) Linear growth rate $\gamma$ (circle markers, blue dashed lines) and real mode frequency $\omega_{r}$ (circle markers, red solid lines) as functions of electron density gradient $a / L_{n e}$ for Case I (c) and Case II (d). Linear growth rate $\gamma$ (diamond markers, green dotted lines) and real mode frequency $\omega_{r}$ (diamond markers, orange dash-dotted lines) for the same cases but with parallel ion motion neglected in GYRO. 

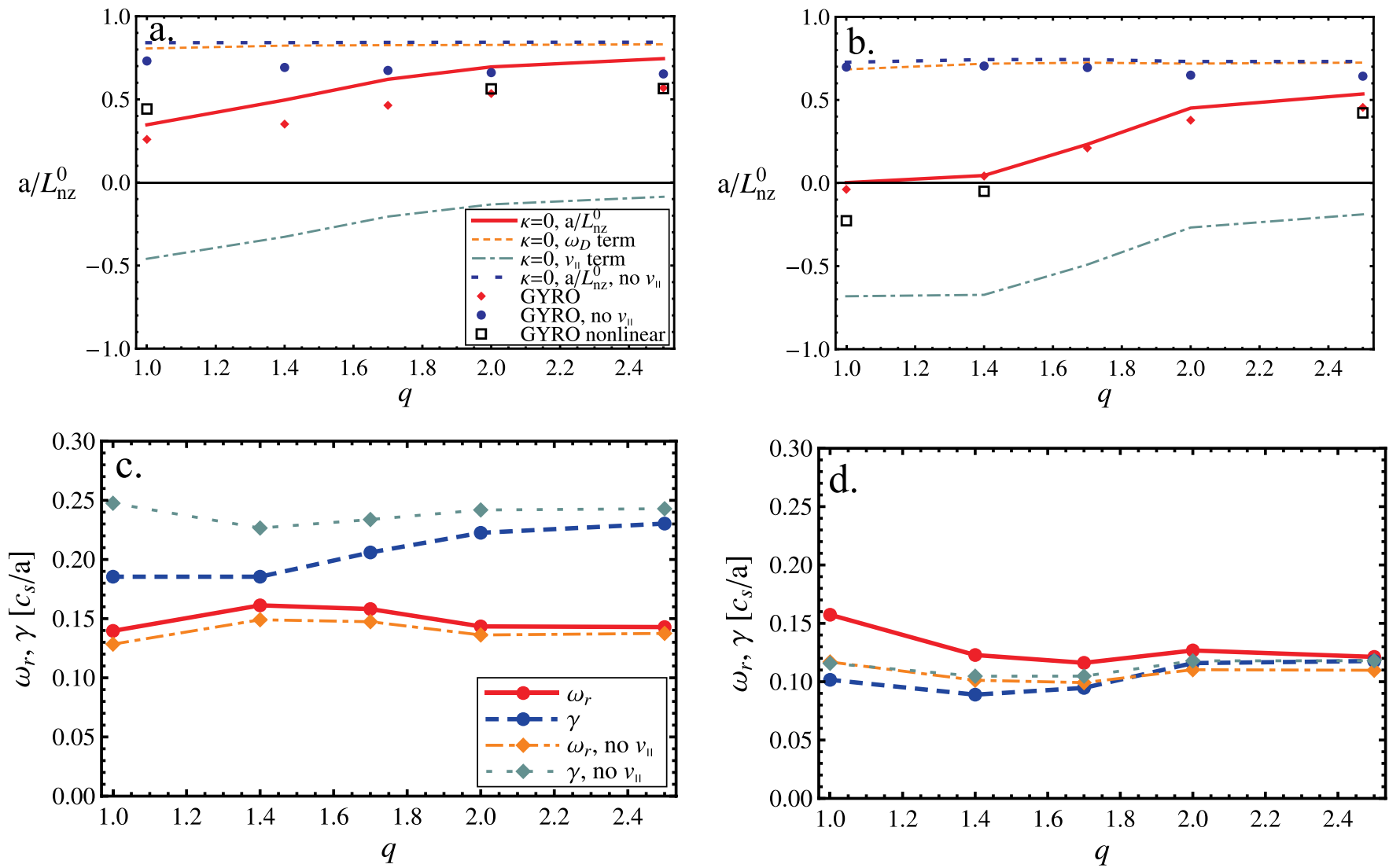

FIG. 8. (a), (b) Impurity peaking factor for trace nickel as function of safety factor $q$ for Case I (a) and Case II (b). Red solid line is the peaking factor from Eq. (5), orange dashed line the magnetic drifts contribution, and green dashed-dotted line the parallel compressibility contribution. Blue dotted line is the peaking factor from Eq. (5) without parallel compressibility effects. Red diamonds and blue dots correspond to GYRo results with and without parallel compressibility effects, respectively, while black hollow squares are results from nonlinear GYRO runs. (c),(d) Linear growth rate $\gamma$ (circle markers, blue dashed lines) and real mode frequency $\omega_{r}$ (circle markers, red solid lines) as functions of safety factor $q$ for Case I (c) and Case II (d). Linear growth rate $\gamma$ (diamond markers, green dotted lines) and real mode frequency $\omega_{r}$ (diamond markers, orange dash-dotted lines) for the same cases but with parallel ion motion neglected in GYRO.

parallel compressibility contribution is significantly more negative with a small safety factor, and as a consequence the peaking factor is more strongly reduced (see Figs. 8(a) and 8(b)). Since the safety factor typically is higher closer to the edge, it could be expected that in TE mode dominated plasmas the impurity parallel dynamics will reduce the peaking factor more and more, the closer to the core we look, while the opposite effect is expected in ITG dominated plasmas, as is confirmed by simulations presented in Ref. 21. However, as discussed in Ref. 5, in a study of collisionless TE modes, it should be noted that the $k_{\theta}$ leading to the largest fluxes is approximately inversely proportional to $q$, which is an effect we miss by keeping $k_{\theta}$ constant in our linear GYRO simulations. Since in the parallel compressibility contribution of Eq. (5) there is a factor $1 /\left(q^{2} k_{\theta}\right)$, it is reduced to $1 / q$ if the mode leading to the largest fluxes should be considered. Furthermore it can also be noted that although the mode frequencies are relatively independent of $q$ (as shown in Figs. $8(\mathrm{c})$ and 8(d)), they are not independent of $k_{\theta} \rho_{s}$ (see Fig. 2) but can on a very crude estimate be expected to vary linearly with $k_{\theta} \rho_{s}$ around the range of $k_{\theta} \rho_{s}$ we analyze. Because of the factor $\omega_{r} /\left(\omega_{r}^{2}+\gamma^{2}\right)$ also found in the parallel compressibility contribution, this would imply that the dependence on $q$ is completely canceled for the linear analysis of the mode leading to the largest fluxes.
Figures 8 (a) and 8 (b) also include impurity peaking factors determined from nonlinear GYRO simulations. These were calculated by linear interpolation of the impurity fluxes from two nonlinear GYRO simulations with different impurity density gradient. In these simulations, to keep an optimal $k_{\theta} \rho_{s}$ resolution around the peak part of the nonlinear energy and particle flux spectra, the spacing between the simulated toroidal mode numbers are changed from case to case while the total number of toroidal modes are held fixed. In the nonlinear simulation for the density gradient driven case we see a significantly weaker, but still existent, $q$-dependence than what the fixed- $k_{\theta}$ linear simulation predicts. This may be understood from the above reasoning about the shift in the peak of the turbulent spectrum. On the other hand, in Case II the trend is found to be similar to the linear predictions, and interestingly the $q$-dependence is even stronger in the nonlinear case.

The difference between including and not-including parallel ion dynamics for lower values of $q$ is more pronounced in the electron temperature gradient driven Case II than in the density gradient driven Case I (a trend, consistent with the results of Ref. 13). This is mostly due to that the factor $\omega_{r} /\left(\omega_{r}^{2}+\gamma^{2}\right)$ in Eq. (5) is larger for Case II than for Case I which can be seen from Figs. 8(c) and 8(d). Accordingly we could expect that for modes where this 
factor is relatively large, the effect of parallel impurity motion on the impurity peaking factor is strengthened. The differences in the dependence on $q$ can only come through the $q$-dependence of $\omega$ and $\phi(\theta)$ since all the other parameters appearing in the third term of Eq. (5) are the same in the two cases for a fixed $q$.

\section{Poloidally asymmetric case}

In earlier studies with a poloidally varying potential present, magnetic shear has been emphasized as one of the most important parameters affecting the impurity peaking. ${ }^{31,34}$ This can be understood from its explicit appearance in the $\mathbf{E} \times \mathbf{B}$ drift term of Eq. (5). Consequently this section will focus on how the impurity peaking factor varies with magnetic shear, and results will be presented for the poloidally asymmetric cases with $\kappa=0.5$ and $\delta=0$ (out-in asymmetry), $\delta=\pi / 2$ (up-down asymmetry), and $\delta=\pi$ (inout asymmetry) in Eq. (1). We will omit the parametric dependence on other parameters in this section because of the structure of the $\mathbf{E} \times \mathbf{B}$ drift term of Eq. (5) and refer to the results of Sec. III B. The inclusion of the $\mathbf{E} \times \mathbf{B}$ drift term typically leads to the addition of a constant to the peaking factor, for other scalings than with $s$. This is because the only way this term can change in these scalings is through $|\phi|^{2}$, and it is not varying by much.
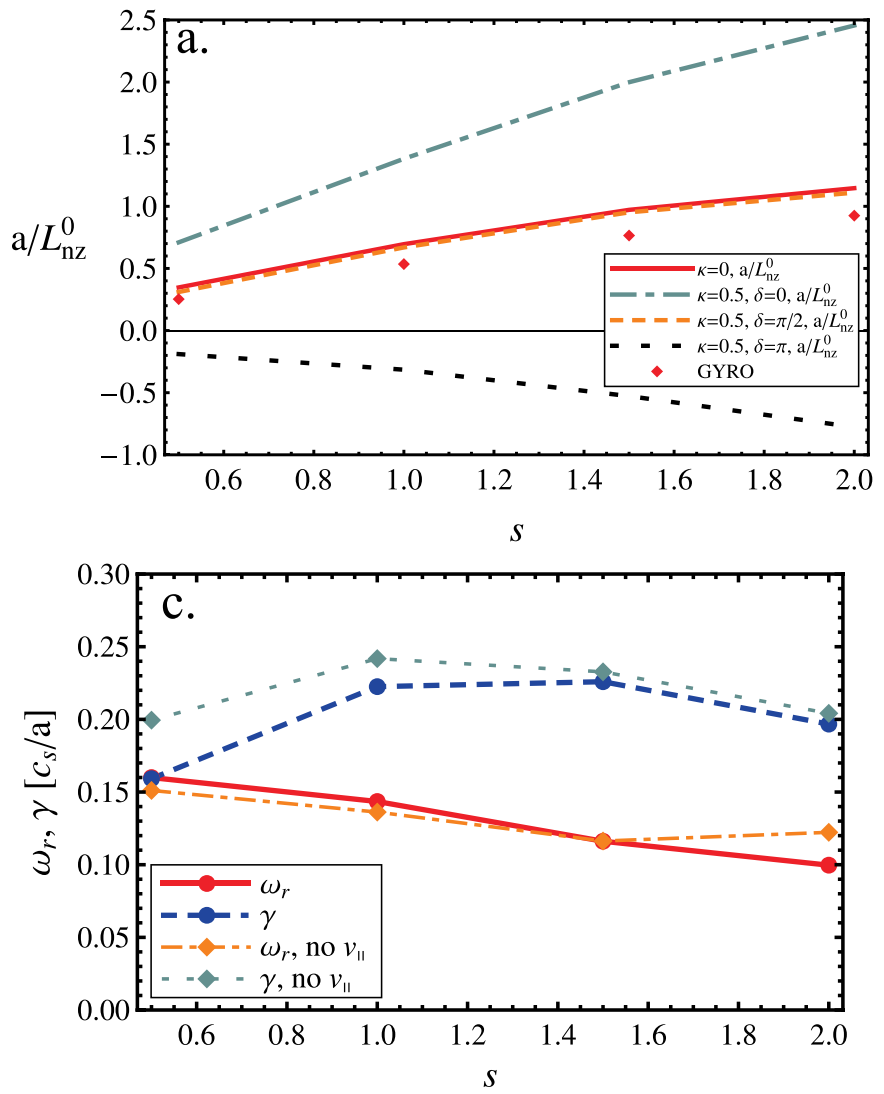

Figures 9(a) and 9(b) show how the peaking factor depends on magnetic shear for Case I and Case II in both the symmetric and asymmetric cases. For Case I the symmetric peaking factor is mainly governed by the contribution from the magnetic drifts, which increases with $s$. The contribution from parallel compressibility is relatively small, and it is not affected much by a change in $s$. When introducing the asymmetry, the peaking factor changes significantly because of the $\mathbf{E} \times \mathbf{B}$ drift term which then is non-zero. In the in-out asymmetric case there is a strong decrease of the impurity peaking, while on the contrary in the out-in asymmetric case there is a strong increase. The peaking factor remains almost unaffected by an up-down asymmetry. Since the $\mathbf{E} \times \mathbf{B}$ drift term becomes larger in magnitude with increasing $s$, the difference between the symmetric and asymmetric peaking factor is also increased with $s$. The reason why the $\mathbf{E} \times \mathbf{B}$ drift term leads to a reduction (an increase) of the peaking factor for inboard (outboard) impurity accumulation is because for $\delta=\pi(\delta=0)$ the term $\langle\theta \sin (\theta-\delta)\rangle_{\phi}$ is negative (positive). Note however that if $s<0$, this term changes sign and leads to an increase (decrease) for inboard (outboard) impurity accumulation, as shown in Ref. 31. The peaking factor in Case II shows a similar behavior to Case I, with the main difference being that the contribution from the parallel compressibility term is significantly larger. Because of this term, the peaking factor can be negative even in the symmetric case.
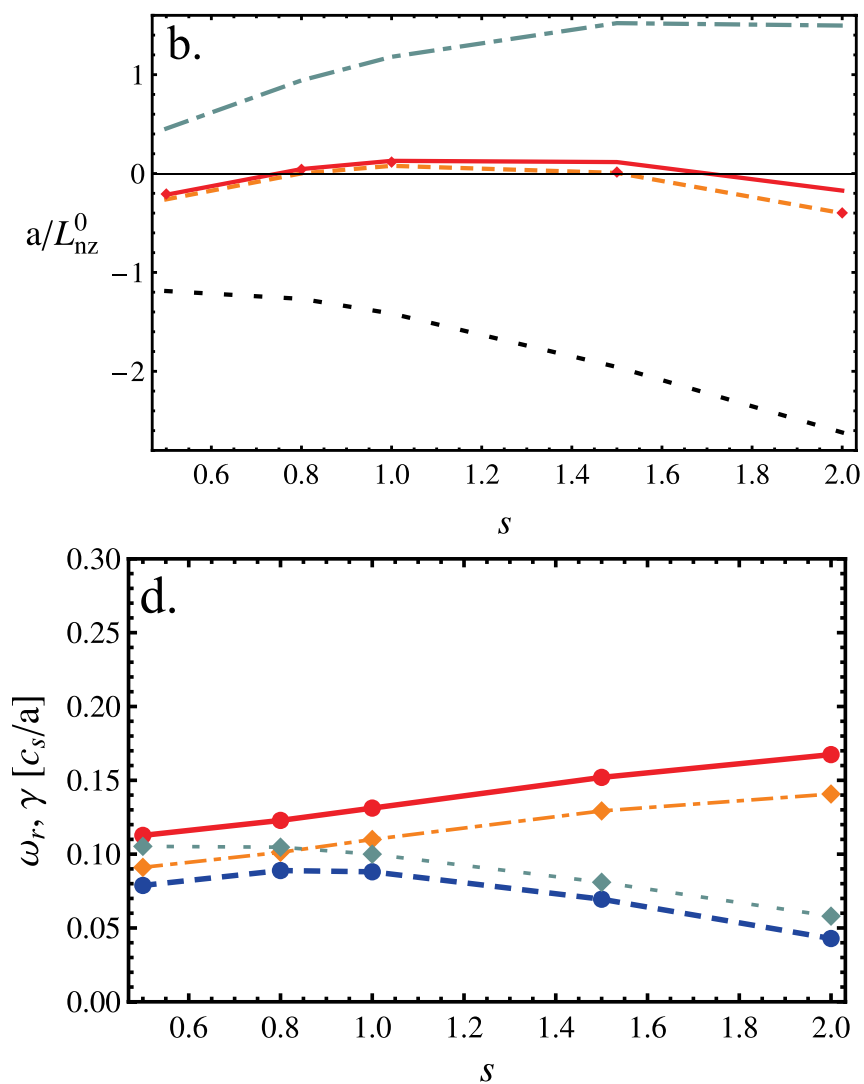

FIG. 9. (a), (b) Impurity peaking factor for trace nickel as function of magnetic shear $s$ for Case I (a) and Case II (b). Red solid line is the peaking factor from Eq. (5) in the symmetric case, green dashed-dotted line corresponds to out-in asymmetry, orange dashed line corresponds to up-down asymmetry, and black dotted line corresponds to in-out asymmetry. Red diamonds correspond to GYRO results. (c),(d) Linear growth rate $\gamma$ (circle markers, blue dashed lines) and real mode frequency $\omega_{r}$ (circle markers, red solid lines) as functions of magnetic shear $s$ for Case I (c) and Case II (d). Linear growth rate $\gamma$ (diamond markers, green dotted lines) and real mode frequency $\omega_{r}$ (diamond markers, orange dash-dotted lines) for the same cases but with parallel ion motion neglected in GYRO. 

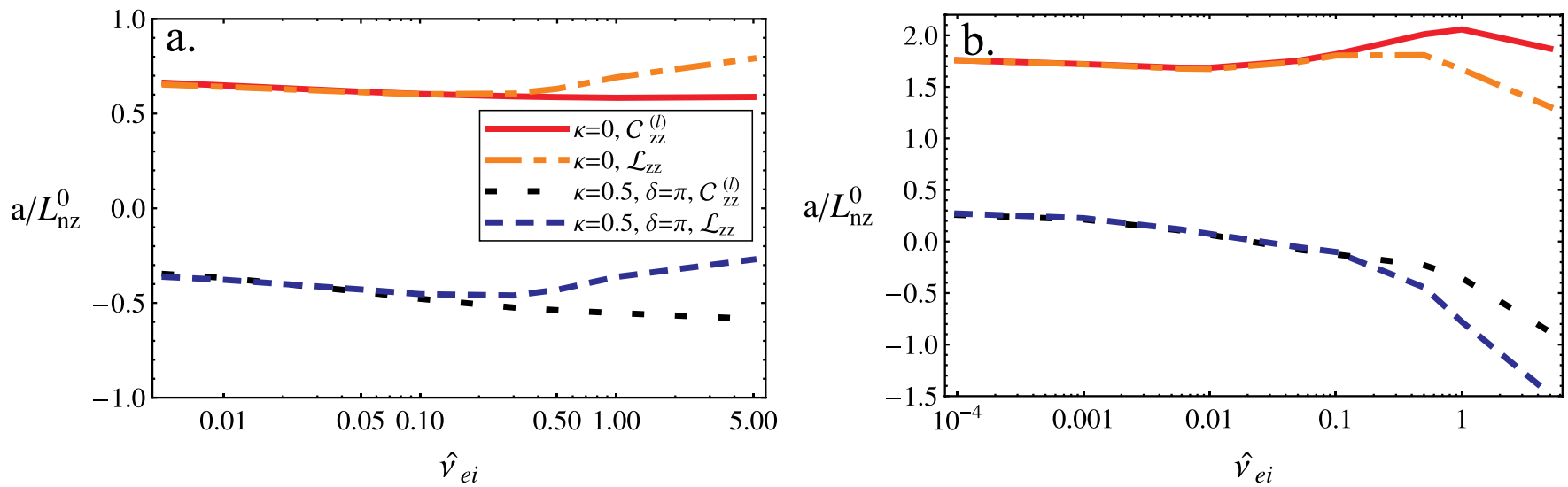

FIG. 10. Impurity peaking factor for trace nickel as function of electron-ion collision frequency $\hat{\nu}_{e i}$ for Case I (a) and an ITG dominated case (b) (note the logarithmic $\hat{\nu}_{e i}$ axis). Red solid line is the peaking factor from Eq. (5) in the symmetric case and black dotted line the corresponding in the in-out asymmetric case. Orange dashed-dotted line is the peaking factor in the symmetric case from a model that utilize the Lorentz collision operator, and blue dashed line the corresponding in the in-out asymmetric case.

Furthermore in Figs. 9(c) and 9(d) it can be noted that the linear eigenvalues are not strongly affected by changes in magnetic shear although for $s \geq 1.0$ there is a small stabilizing effect with increasing shear. Reference 44 reported on a stabilizing effect which was weaker if the density gradients were large. This is consistent with what is observed here since Case I has a larger density gradient than Case II and the stabilizing effect is weaker in Case I. An increasing positive magnetic shear can stabilize a TE mode through FLR effects, but it can also drive the mode more unstable by increasing bad magnetic curvature. The dependence of linear growth rate on shear is consequently not trivial. The eigenvalues do not change much if the parallel ion dynamics is neglected.

\section{Collisions}

The model represented by Eq. (5) models impurity selfcollisions by the full linearized impurity-impurity collision operator $C_{z z}^{(l)}$, and it is found that up to the considered order, $\mathcal{O}\left(Z^{-1}\right)$, the effect of collisions does not appear explicitly. It thus predicts that the only way for collisions to affect the impurity peaking is through their impact on the mode characteristics. This leads to a conclusion that in reality, collisions should have a relatively weak influence on the impurity peaking factor. As many of the easily accessible gyrokinetic tools employ non-momentum-conserving model operators, it is interesting to see whether or not the form of the collision operator affects the above result.

In Appendix we present an alternative model which uses the Lorentz (or "pitch-angle scattering") operator to model impurity self-collisions but is otherwise similar to the perturbative solution represented by Eq. (5). The most striking difference between the two models is the appearance of the factor $1 /\left(1+i \nu_{D}(x) / \omega\right)$ in the contribution related to parallel dynamics, where $\nu_{D}(x)\left[\propto \hat{\nu}_{e i}\right]$ is the deflection frequency. This implies that in the case of the Lorentz operator the effect of collisions appears explicitly in the expression for the impurity peaking factor, which is an artifact of $C_{z z}\left[v_{\|} f_{z 0}\right]$ being different from zero. Figure 10 shows a comparison between the two models for Case I, but also for an ITG dominated case (earlier studied in Ref. 34 also using fully ionized trace Nickel with local profile and geometry parameters: $r / a=0.3, R_{0} / a=3, k_{\theta} \rho_{s}=0.3, q=1.7, s=1.5, a / L_{n e}$ $=1.5, T_{i} / T_{e}=0.85, a / L_{T e}=2$, and $a / L_{T i}=2.5$ ). Case II is not considered since it is stabilized already at low collisionality as shown in Fig. 3. The scalings illustrate that the impact of the Lorentz collisions starts to become important for $\hat{\nu}_{e i} \gtrsim 0.1(\sim|\omega|)$, and the two models start to diverge. The use of the Lorentz operator leads to an overestimation of the impurity peaking factor in the TE mode case, when the collision frequency is high, both in the poloidally symmetric case as well as in the asymmetric case. On the contrary, in the ITG mode case the use of the Lorentz operator leads to an underestimation of the peaking factor. This is expected because of the impact of $1 /\left(1+i \nu_{D}(x) / \omega\right)$ in the parallel dynamics term, which decreased in magnitude with increasing $\nu_{D}(x)$. Since parallel dynamics decreases (increases) the peaking factor for TE (ITG) modes we find an increase (a decrease) in the peaking factor with increasing $\nu_{D}(x)$.

\section{CONCLUSIONS}

The paper presents a quasilinear study of two collisionless TE mode cases, driven by the density gradient and the electron temperature gradient, respectively, including their mode characteristics and their effect on impurity transport. Mode characteristics have been obtained by linear gyrokinetic simulations using GYRO. The poloidal wave number was chosen as $k_{\theta} \rho_{s}=0.15$ for both cases to represent the mode with the largest fluxes in nonlinear simulations. In agreement with previous studies, the electron temperature gradient driven mode is suppressed for small collisionalities, while the density gradient driven mode not only remains unstable even for very high collision frequencies. We also observe its transition to a dissipative TE mode. The dependence of growth rate and real frequency on safety factor and magnetic shear is nonmonotonic and within small variations.

To investigate the peaking of high- $Z$ trace impurities in tokamak plasmas we use an approximate gyrokinetic model and compare it to results obtained with GYRO. It is observed that parameters such as $T_{i} / T_{e}, a / L_{T e}$ and $a / L_{n e}$ mainly affect the peaking through their impact on mode characteristics, 
particularly, the factor $\omega_{r} /\left(\omega_{r}^{2}+\gamma^{2}\right)$ is important to determine the effect of the impurity parallel dynamics. As noted before, in fluid modeling this factor enters directly into the parallel compressibility term of the approximate model and is consequently responsible for determining the size of this contribution. Parameters describing the magnetic geometry, $q$ and $s$, have a more significant influence on the peaking because of their explicit appearance in certain contributions. An increase in magnetic shear typically leads to an increase of the impurity peaking factor in the poloidally symmetric case because of the increase in the magnetic drift contribution. However in the poloidally asymmetric case since the term describing the $\mathbf{E} \times \mathbf{B}$ drift of impurities in the nonfluctuating electrostatic potential has an explicit linear shear dependence, an increase in shear can lead to a significant reduction or enhancement of the impurity peaking, depending on the location of the potential minimum. Increasing safety factor leads to a decrease of the relative significance of the impurity parallel dynamics contribution, but the effect on the peaking depends on the sign of $\omega_{r}$, and for TE modes, with $\omega_{r}>0$, it results in an increase of the peaking factor. Nonlinear simulations in the density gradient driven TE case show only a very weak $q$ scaling. This can be explained by a nonlinear shift in the poloidal wave number $\left(k_{\theta} \sim 1 / q\right.$, as shown in Ref. 5). However, in the temperature gradient driven TE case, the $q$ scaling is even stronger non-linearly than the fixed $k_{\theta}$ linear modeling predicts.

The model using the conservation properties of the full linearized collision operator for impurity self collisions show that collisions can only indirectly affect impurity transport through changes in the mode characteristics. We show that when a non-momentum-conserving model operator is used, such as a Lorentz operator, the parallel compressibility contribution to the peaking factor is modified leading to errors in the collisionality dependence. This effect becomes important when the impurity collision frequency becomes comparable to the mode frequency.

\section{ACKNOWLEDGMENTS}

This work was funded by the European Communities under Association Contract between EURATOM and Vetenskapsrådet. The views and opinions expressed herein do not necessarily reflect those of the European Commission. The simulations were performed on resources provided by the Swedish National Infrastructure for Computing (SNIC) at PDC Center for High Performance Computing (PDC-HPC), and on the HPC-FF cluster at the Jülich Supercomputing Center (JSC). The authors would like to thank J. Candy for providing the GYRO code and Ye. O. Kazakov for fruitful discussions.

\section{APPENDIX: DERIVATION OF PEAKING FACTOR USING A LORENTZ COLLISION OPERATOR}

In this section we derive a model for the impurity peaking factor similar to that represented by Eq. (5), but using the Lorentz collision operator instead of the linearized impurityimpurity collision operator. We neglect $\mathcal{O}(\epsilon)$ corrections.
The Lorentz collision operator for impurity selfcollisions is given by

$$
C\left(g_{z}\right)=\frac{\nu_{D}(x)}{2} \mathcal{L}\left(g_{z}\right) \equiv \frac{\nu_{D}(x)}{2} \frac{\partial}{\partial \xi}\left[\left(1-\xi^{2}\right) \frac{\partial g_{z}}{\partial \xi}\right],
$$

where $\nu_{D}$ is the deflection frequency for self-collisions $\nu_{D}(x)=\hat{\nu}_{z z}[\operatorname{Erf}(x)-G(x)] / x^{3}, \quad \hat{\nu}_{z z}=n_{z} Z^{4} e^{4} \ln \Lambda /\left[4 \pi \epsilon_{0}^{2} m_{z}^{1 / 2}\right.$ $\left.\left(2 T_{z}\right)^{3 / 2}\right]$, and $\ln \Lambda$ is the Coulomb logarithm. $\operatorname{Erf}(\mathrm{x})$ is the error-function and $G(x)=\left[\operatorname{Erf}(x)-x \operatorname{Erf}^{\prime}(x)\right] /\left(2 x^{2}\right)$ the Chandrasekhar function. In the Lorentz operator $\xi=x_{\|} / x$ denotes the cosine of the pitch-angle.

We assume the ordering $\omega_{D z} / \omega \sim \omega_{* z}^{T} / \omega \sim \omega_{E} / \omega \sim$ $J_{0}\left(z_{z}\right)-1 \sim 1 / Z$ and expand $g_{z}$ in $1 / \sqrt{Z}$ keeping terms up to $\mathcal{O}\left(Z^{-1}\right)$, i.e., $g_{z} \approx g_{0}+g_{1}+g_{2}$. The 0 th order solution of GK equation (3) is

$$
g_{0}=\frac{Z e \phi f_{z 0}}{T_{z}}
$$

with $C\left[g_{0}\right]=0$. This, added to the adiabatic response, $-Z e \phi f_{z 0} / T_{z}$, merely tells that the impurities are so heavy and bound to the field lines through their high charge, that they do not respond to electrostatic fluctuations to lowest order in $1 / Z$. This justifies neglecting the effect of impurities on the mode characteristics, in spite of our assumption $n_{z} Z^{2} / n_{e} \sim 1$ (that is required to make self-collisions dominate).

The 1st order GK equation reads

$$
\frac{v_{\|}}{q R} \frac{\partial g_{0}}{\partial \theta}-i \omega g_{1}-C\left[g_{1}\right]=0
$$

and is solved by assuming that the solution can be written in terms of Legendre polynomials $P_{n}(\xi)$ as $g_{1}=g_{1}^{0}(x) P_{0}(\xi)$ $+g_{1}^{1}(x) P_{1}(\xi)$. Here $P_{0}(\xi)=1, P_{1}(\xi)=\xi$ and we remind about the properties $\int_{-1}^{1} d \xi P_{n}(\xi) P_{m}(\xi)=2 \delta_{m n} /(2 n+1)$ and $\mathcal{L}\left[P_{n}(\xi)\right]=-n(n+1) P_{n}(\xi)$. The solution to Eq. (A3) is found to be

$$
g_{1}=-i \frac{v_{\|}}{q R} \frac{Z e f_{z 0}}{T_{z}} \frac{\partial \phi}{\partial \theta} \frac{1}{\omega} \frac{1}{1+i \nu_{D} / \omega} .
$$

Note that for a momentum conserving collision operator $C\left[g_{1} \propto v_{||} f_{z 0}\right]=0$ in Eq. (A3); thus, $\nu_{D}$ would not appear in Eq. (A4).

The 2 nd order GK equation is

$$
i \omega g_{2}+C\left[g_{2}\right]=i \omega_{D z} g_{0}+\frac{v_{\|}}{q R} \frac{\partial g_{1}}{\partial \theta}-i \frac{Z e f_{z 0}}{T_{z}} \phi\left(\omega_{* z}^{T}+\omega \frac{z_{z}^{2}}{4}\right) .
$$

The velocity anisotropies enter in $\omega_{D z}$ and $z_{z}$, which we can rewrite in terms of Legendre polynomials as $\omega_{D z} \equiv$ $(1 / 3)\left[2 P_{0}(\xi)+P_{2}(\xi)\right] \omega_{D x}$ and $z_{z}^{2} \equiv(2 / 3)\left[P_{0}(\xi)-P_{2}(\xi)\right] z_{x}^{2}$, where $P_{2}(\xi)=\left(3 \xi^{2}-1\right) / 2$, and $\omega_{D x}=\omega_{D x}(x), z_{x}=z_{x}(x)$ only depend on speed. Furthermore, by noting that $\partial \mathcal{E} / \partial \theta=$ 0 (where $\mathcal{E}=m_{z} v^{2} / 2+Z e \phi_{E}$ ) we can find the identity

$$
v_{\|} \frac{\partial v_{\|}}{\partial \theta}=\frac{1}{3}\left(P_{2}(\xi)-P_{0}(\xi)\right) v^{2} \frac{\partial \ln B}{\partial \theta}-\frac{Z e}{m_{z}} \frac{\partial \phi_{E}}{\partial \theta} P_{0}(\xi) .
$$


We now search for a solution to Eq. (A5) of the form

$$
g_{2}=g_{2}^{0}(x) P_{0}(\xi)+g_{2}^{2}(x) P_{2}(\xi),
$$

where we realize that $g_{2}^{2}$ will not contribute to the particle flux since $\int_{-\infty}^{\infty} d \xi P_{2}(\xi)=0$. By substituting Eq. (A7) into Eq. (A5) and collecting the parts proportional to $P_{0}$ we find that

$$
\begin{aligned}
g_{2}^{0}= & \frac{Z e \phi}{T_{z}} f_{z 0}\left[\frac{2}{3} \frac{\omega_{D x}}{\omega}-\frac{z_{x}^{2}}{6}-\frac{\omega_{* z}^{T}}{\omega}+\frac{\omega_{E}}{\omega}\right] \\
& +\frac{1}{q^{2} R^{2}} f_{z 0} \frac{Z e}{T_{z}} \frac{\partial \phi}{\partial \theta} \frac{1}{\omega^{2}} \frac{1}{1+i \nu_{D} / \omega} \frac{Z e}{m_{z}} \frac{\partial \phi_{E}}{\partial \theta} \\
& -\frac{Z e}{T_{z}} \frac{f_{z 0}}{q^{2} R} \frac{v^{2}}{3 \omega^{2}} \frac{1}{1+i \nu_{D} / \omega}\left[\frac{\partial}{\partial \theta}\left(\frac{1}{R} \frac{\partial \phi}{\partial \theta}\right)-\frac{1}{R} \frac{\partial \phi}{\partial \theta} \frac{\partial \ln B}{\partial \theta}\right] .
\end{aligned}
$$

From $g \approx g_{0}+g_{1}+g_{2}$, only the $g_{2}^{0}$ part of $g_{2}$ contributes to the particle flux; thus, the impurity peaking factor is found from solving

$$
\begin{aligned}
0 & =\left\langle\Gamma_{z}\right\rangle=-\left\langle\frac{k_{\theta}}{B} \Im\left[\int d^{3} v J_{0}\left(z_{z}\right) g_{z} \phi^{*}\right]\right\rangle \\
& \approx-\left\langle\frac{k_{\theta}}{B} \Im\left\{\int d^{3} v g_{2}^{0} \phi^{*}\right\}\right\rangle,
\end{aligned}
$$

where higher order than $1 / Z$ corrections to the impurity flux are neglected.

${ }^{1}$ D. R. Ernst, P. T. Bonoli, P. J. Catto, W. Dorland, C. L. Fiore, R. S. Granetz, M. Greenwald, A. E. Hubbard, M. Porkolab, M. H. Redi, J. E. Rice, K. Zhurovich, and Alcator C-Mod Group, Phys. Plasmas 11, 2637 (2004).

${ }^{2}$ B. B. Kadomtsev and O. P. Pogutse, Sov. Phys. JETP 24, 1172 (1967).

${ }^{3}$ M. Kikuchi, K. Lackner, and M. Q. Tran, Fusion Physics (IAEA, 2012),

Sec. 2.3.4.3. See http://www-pub.iaea.org/books/IAEABooks/8879/ Fusion-Physics.

${ }^{4}$ C. Estrada-Mila, J. Candy, and R. E. Waltz, Phys. Plasmas 12, 022305 (2005).

${ }^{5}$ T. Dannert and F. Jenko, Phys. Plasmas 12, 072309 (2005).

${ }^{6}$ A. Casati, C. Bourdelle, X. Garbet, F. Imbeaux, J. Candy, F. Clairet, G. Dif-Pradalier, G. Falchetto, T. Gerbaud, V. Grandgirard, O. D. Gürcan, P. Hennequin, J. Kinsey, M. Ottaviani, R. Sabot, Y. Sarazin, L. Vermare, and R. E. Waltz, Nucl. Fusion 49, 085012 (2009).

${ }^{7}$ C. Angioni and A. G. Peeters, Phys. Rev. Lett. 96, 095003 (2006).

${ }^{8}$ C. Angioni, R. Dux, E. Fable, A. G. Peeters, and ASDEX Upgrade Team, Plasma Phys. Controlled Fusion 49, 2027 (2007).

${ }^{9}$ M. E. Puiatti, M. Valisa, C. Angioni, L. Garzotti, P. Mantica, M. Mattioli, L. Carraro, I. Coffey, C. Sozzi, and JET-EFDA Contributors, Phys. Plasmas 13, 042501 (2006).

${ }^{10}$ T. Fülöp and J. Weiland, Phys. Plasmas 13, 112504 (2006).

${ }^{11}$ T. Fülöp and H. Nordman, Phys. Plasmas 16, 032306 (2009).

${ }^{12}$ T. Fülöp, S. Braun, and I. Pusztai, Phys. Plasmas 17, 062501 (2010).

${ }^{13}$ I. Pusztai, S. Moradi, T. Fülöp, and N. Timchenko, Phys. Plasmas 18, 082506 (2011).

${ }^{14}$ S. Moradi, M. Z. Tokar, and B. Weyssow, Phys. Plasmas 17, 012101 (2010).

${ }^{15}$ C. Bourdelle, X. Garbet, F. Imbeaux, A. Casati, N. Dubuit, R. Guirlet, and T. Parisot, Phys. Plasmas 14, 112501 (2007).

${ }^{16}$ T. Parisot, R. Guirlet, C. Bourdelle, X. Garbet, N. Dubuit, F. Imbeaux, and P. R. Thomas, Plasma Phys. Controlled Fusion 50, 055010 (2008).

${ }^{17}$ D. Villegas, R. Guirlet, C. Bourdelle, G. T. Hoang, X. Garbet, and R. Sabot, Phys. Rev. Lett. 105, 035002 (2010).
${ }^{18}$ S. Futatani, X. Garbet, S. Benkadda, and N. Dubuit, Phys. Rev. Lett. 104, 015003 (2010).

${ }^{19}$ N. Dubuit, X. Garbet, T. Parisot, R. Guirlet, and C. Bourdelle, Phys. Plasmas 14, 042301 (2007).

${ }^{20}$ A. Skyman, H. Nordman, and P. Strand, Phys. Plasmas 19, 032313 (2012).

${ }^{21}$ N. T. Howard, M. Greenwald, D. R. Mikkelsen, M. L. Reinke, A. E. White, D. Ernst, Y. Podpaly, and J. Candy, Nucl. Fusion 52, 063002 (2012).

${ }^{22}$ H. Nordman, A. Skyman, P. Strand, C. Giroud, F. Jenko, F. Merz, V. Naulin, T. Tala, and JET-EFDA Contributors, Plasma Phys. Controlled Fusion 53, 105005 (2011).

${ }^{23}$ C. Angioni, A. G. Peeters, G. V. Pereverzev, A. Bottino, J. Candy, R. Dux, E. Fable, T. Hein, and R. E. Waltz, Nucl. Fusion 49, 055013 (2009).

${ }^{24}$ R. Neu, R. Dux, A. Geier, H. Greuner, K. Krieger, H. Maier, R. Pugno, V. Rohde, S. W. Yoon, and ASDEX Upgrade Team, J. Nucl. Mater. 313-316, 116 (2003).

${ }^{25}$ R. Dux, R. Neu, A. G. Peeters, G. Pereverzev, A. Mück, F. Ryter, J. Stober, and ASDEX Upgrade Team, Plasma Phys. Controlled Fusion 45, 1815 (2003).

${ }^{26}$ M. Valisa, L. Carraro, I. Predebon, M. E. Puiatti, C. Angioni, I. Coffey, C. Giroud, L. L. Taroni, B. Alper, M. Baruzzo, P. B. daSilva, P. Buratti, L. Garzotti, D. Van Eester, E. Lerche, P. Mantica, V. Naulin, T. Tala, M. Tsalas, and JET-EFDA Contributors, Nucl. Fusion 51, 033002 (2011).

${ }^{27}$ M. E. Puiatti, M. Valisa, M. Mattioli, T. Bolzonella, A. Bortolon, I. Coffey, R. Dux, M. von Hellermann, P. Monier-Garbet, M. F. F. Nave, J. Ongena, and Contributors to the EFDA-JET Workprogramme, Plasma Phys. Controlled Fusion 45, 2011 (2003).

${ }^{28}$ C. Giroud, C. Angioni, G. Bonheure, I. Coffey, N. Dubuit, X. Garbet, R. Guirlet, P. Mantica, V. Naulin, M. E. Puiatti, M. Valisa, A. D. Whiteford, K.-D. Zastrow, M. N. A. Beurskens, M. Brix, E. de la Luna, K. Lawson, L. Lauro-Taroni, A. Meigs, M. O'Mullane, T. Parisot, C. P. Von Thun, O. Zimmermann, and JET-EFDA Contributors, in Proceedings of 21 st International Conference on Fusion Energy, 2006, Chengdu, China, 2006 (IAEA, Vienna).

${ }^{29}$ T. Fülöp and S. Moradi, Phys. Plasmas 18, 030703 (2011).

${ }^{30}$ S. Moradi, T. Fülöp, A. Mollén, and I. Pusztai, Plasma Phys. Controlled Fusion 53, 115008 (2011).

${ }^{31}$ A. Mollén, I. Pusztai, T. Fülöp, Ye. O. Kazakov, and S. Moradi, Phys. Plasmas 19, 052307 (2012).

${ }^{32}$ M. L. Reinke, I. H. Hutchinson, J. E. Rice, N. T. Howard, A. Bader, S. Wukitch, Y. Lin, D. C. Pace, A. Hubbard, J. W. Hughes, and Y. Podpaly, Plasma Phys. Controlled Fusion 54, 045004 (2012).

${ }^{33}$ J. Candy and R. E. Waltz, J. Comput. Phys. 186, 545 (2003).

${ }^{34}$ I. Pusztai, A. Mollén, T. Fülöp, and J. Candy, "Turbulent transport of impurities and their effect on energy confinement," Plasma Phys. Controlled Fusion (to be published).

${ }^{35}$ GYRO nonlinear gyrokinetic simulation database https://github.com/gafusion/doc/raw/master/database/gyro-database.pdf.

${ }^{36}$ E. Belli and J. Candy, Plasma Phys. Controlled Fusion 50, 095010 (2008).

${ }^{37}$ C. Angioni, A. G. Peeters, F. Jenko, and T. Dannert, Phys. Plasmas 12 112310 (2005).

${ }^{38}$ C. Angioni, R. M. McDermott, E. Fable, R. Fischer, T. Pütterich, F. Ryter, G. Tardini, and ASDEX Upgrade Team, Nucl. Fusion 51, 023006 (2011).

${ }^{39}$ C. Angioni, F. J. Casson, C. Veth, and A. G. Peeters, Phys. Plasmas 19, 122311 (2012)

${ }^{40}$ Y. Camenen, A. G. Peeters, C. Angioni, F. J. Casson, W. A. Hornsby, A. P. Snodin, and D. Strintzi, Phys. Plasmas 16, 012503 (2009).

${ }^{41}$ F. J. Casson, A. G. Peeters, C. Angioni, Y. Camenen, W. A. Hornsby, A. P. Snodin, and G. Szepesi, Phys. Plasmas 17, 102305 (2010).

${ }^{42}$ L. C. Ingesson, H. Chen, P. Helander, and M. J. Mantsinen, Plasma Phys. Controlled Fusion 42, 161 (2000).

${ }^{43}$ Ye. O. Kazakov, I. Pusztai, T. Fülöp, and T. Johnson, Plasma Phys. Controlled Fusion 54, 105010 (2012).

${ }^{44}$ J. Lang, Y. Chen, and S. E. Parker, Phys. Plasmas 14, 082315 (2007).

${ }^{45}$ A. G. Peeters, C. Angioni, M. Apostoliceanu, F. Jenko, F. Ryter, and ASDEX Upgrade Team, Phys. Plasmas 12, 022505 (2005).

${ }^{46}$ A. Skyman, H. Nordman, and P. I. Strand, Nucl. Fusion 52, 114015 (2012).

${ }^{47}$ See http://www.ipp.mpg.de/ fsj/gene/ for GENE. 